\title{
The only one strategy in context
}

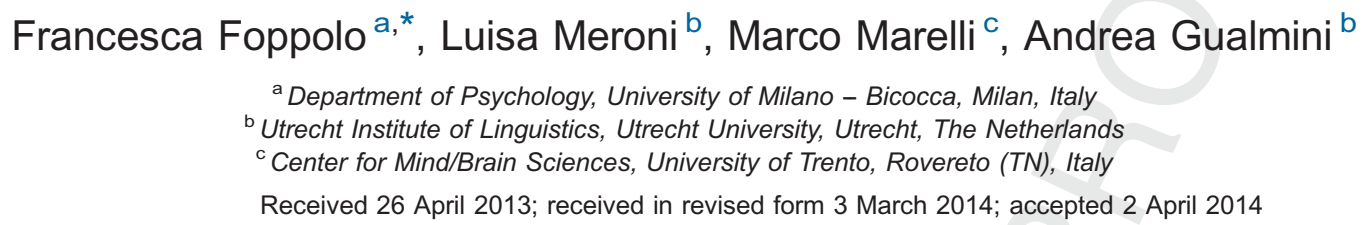

\section{Abstract}

This paper investigates domain restriction in the resolution of privative ambiguities of sentences like The orange parakeet is the only one that is hiding itself, which is ambiguous between an anaphoric and an exophoric interpretation. Previous work by Crain et al. (1994) argued for a built-in parsing preference for weak readings of privative ambiguities. Manipulating the amount of the contextual information available, we present results that challenge Crain et al.'s conclusion: in our study, we show that context takes precedence over truthQ2 conditional considerations in the resolution of privative ambiguities in adults.

(C) 2014 Published by Elsevier B.V.

\section{Introduction}

In this paper we investigate how adults resolve a special case of semantic ambiguity, i.e. privative ambiguity (Horn, 1989; Zwicky and Sadock, 1975). We start from the pioneering study by Crain et al. (1994), who tested the phenomenon of "one-substitution" in children and adults by investigating sentences like (1):

(1) The big elephant is the only one that is playing guitar.

Sentence (1) contains the expression "the only one", which needs a referent to be interpreted. This gives rise to an ambiguity. The ambiguity derives from the fact that one can be interpreted anaphorically, as in (2a), or exophorically, as in (2b):

$(2)$

a. The big elephant is the only elephant that is playing guitar. (anaphoric interpretation)

b. The big elephant is the only animal that is playing guitar. (exophoric interpretation)

In (2a), one receives its reference from the linguistic antecedent that is found in the head noun of the subject Determiner Phrase (DP henceforth), i.e. elephant in this case. In (2b), one is interpreted by making reference to a wider

\footnotetext{
* Corresponding author at: Department of Psychology, University of Milano - Bicocca, P.zza dell'Ateneo Nuovo 1, ed. U6/3136, 20126 Milano, Italy. Tel.: +3902 64483788 .

E-mail address: francesca.foppolo@unimib.it (F. Foppolo).
} 
discourse domain that, besides elephants, might include other animals or individuals. Crucially, an entailment relation occurs between the alternative readings in (2), and this is what makes the present ambiguity a privative one. In our example, the exophoric interpretation (2b) asymmetrically entails the anaphoric interpretation (2a). This means that, whenever (1) is true under interpretation (2a), it would also be true under interpretation (2b), but not vice versa. To illustrate, if it is true that there is only one animal in the discourse domain that is doing $\mathrm{P}$ (exophoric interpretation, (2b)), then it follows that no other elephant (except for the big one) is doing $\mathrm{P}$ as well. By contrast, if it is true that there is no other elephant (except for the big one), that is doing $\mathrm{P}$ (anaphoric interpretation, (2a)), then there might be an animal of a different species in the discourse domain that is doing $\mathrm{P}$ as well. Consistent with the semantic literature, we can say that (2a) is the weak interpretation, i.e. the one that requires fewer commitments about the status of the world in which a sentence like (1) is uttered: by interpreting (1) as (2a) one will only commit to the fact that no other elephant, except for the big one, is playing the guitar, without making any claim about any other animal that might be present in the discourse domain.

Other linguistic phenomena exhibit the same pattern of entailment between alternative readings and have been the source of intense semantic theorizing. For example, the sentence "The girls know each other" might be interpreted as "Every girl knows every other girl" or "Some girls know some other girls", the former reading being generally preferred (subject to the lexical properties of the items it combines with). Analogously to what happens in case of "the only one", the alternative interpretations of "each other" spelled out above are not truth-conditionally independent, in that one reading entails the other. Our alleged preference for the strong interpretation of reciprocals has been accounted for in terms of the Strongest Meaning Hypothesis proposed by Dalrymple et al. (1998) and subsequently extended to plural predication by Winter (2001). In a similar vein, Krifka (1998) proposed the Principle of Pragmatic Strengthening to account for our preferences in the resolution of plural predication and the so-called donkey sentences (i.e. sentences like "Every farmer who owns a donkey beats it") in which, analogously, an entailment relation occurs between alternative readings (every farmer who owns a donkey beats all/some of the donkeys he owns). This principle assumes that, whenever alternative readings are allowed by the grammar, we should favour the stronger over the weaker one (if consistent with general background assumptions). A similar argument is also implemented in Chierchia (2006) to account for the interpretation of sentences involving scalar terms like some and polarity sensitive items like everand any, whose interpretation involves considerations about informativeness (see also Chierchia et al., 2013). For example, the interpretation "Lyn ate some but not all of the cookies" is more informative than "Lyn ate some cookies" in upward entailing contexts (Ladusaw, 1980) and thus a sentence such as 'Lyn ate some cookies" is pragmatically enriched and most often interpreted as "Lyn ate some but not all of the cookies". This some-but-not all inference does not arise in downward entailing contexts, in which the at-least-some meaning is the strongest interpretation and thus the one generally preferred (e.g., consider the conditional sentence "If Lyn ate some of the cookies, she'll feel sick", that does not convey the inference that Lyn will not feel sick if she eats all of the cookies). Analogously, considerations about the informativeness of alternative representations are central in theoretical accounts of presupposition projection (cf. Geurts, 2000 for a criticism and references therein) and in the analysis of scope interactions (cf. Gualmini et al., 2008 and references therein).

In this paper, we restrict our attention to the case of sentences like (1) that were investigated by Crain et al. (1994) in two experiments. In a first experiment, Crain and colleagues tested 3-5 year-old English speaking children with a Truth Value Judgement task (Crain and McKee, 1985). They asked children to evaluate sentences like (1) in a context in which (1) was true under the anaphoric interpretation (2a) but false under the exophoric interpretation (2b). In the scenarios used for the evaluation, the big elephant was the only elephant playing a guitar, but not the only animal that was doing it, since an octopus and a bird were also playing a guitar. The authors found that children consistently rejected the target sentences in this scenario. To motivate their answer, children pointed to the other animal(s) that were doing the same action as the subject DP in the target sentences. Crain and colleagues interpreted children's behaviour by appealing to a Maximal Commitment strategy: when interpreting ambiguous sentences, children would initially select the interpretation that has more chances of being falsified. In the case at hand, children would interpret (1) by selecting the strong, exophoric, interpretation (2b). This result was explained by appealing to the Semantic Subset Principle (Crain, 1992, 1993; Crain and Philip, 1993), according to which, in order to converge on the target grammar, the first hypothesis that children make in solving semantic ambiguities is the one that can be falsified on the basis of positive evidence from the input (for a criticism, see also Gualmini and Schwarz, 2009; Musolino, 2006).

In a second experiment, Crain et al. tested a group of adults with a sentence falsification task in which participants were given sentences like (1) and were asked to imagine a scenario that made those sentences false. What they found is that adults tended to mention an animal of the same individual-level category as the head noun of the subject DP. In the case of (1), for example, adults imagined a situation in which there was another elephant that was playing a guitar too (besides the big elephant), thereby suggesting that (1) was disambiguated anaphorically, as in (2a). Crain et al. interpreted adults' results in terms of the Principle of Parsimony: "if there is a reading that carries fewer unsatisfied presuppositions or entailments than any other, then, other criteria of plausibility being equal, that reading will be adopted as most plausible by 
the hearer, and the presuppositions in question will be incorporated in his or her model"' (Crain and Steedman, 1985: 333). This principle was ultimately motivated by the need to minimize cognitive effort. On this basis, Crain and colleagues hypothesized that the adult parser follows a strategy of Minimal Commitment: in order to avoid unnecessary commitments that might need to be changed at a later stage, adult speakers prefer the interpretation that has more chances of being true. This means that, when interpreting ambiguous sentences in which alternative readings stand in a subset-superset relation, as it is the case for (1), the parser would prefer the weak interpretation, the one that is true in a wider set of circumstances. For example, (1) under the anaphoric interpretation (2a) would be true in the scenario presented to children, in which other animals (besides elephants) were performing the same action as the big elephant. In the same situation, the exophoric interpretation $(2 b)$ would be false.

Let us sum up. On the basis of their findings, Crain et al. proposed the following generalizations about the adult parser: first, it is claimed to be parsimonious, in that it selects the interpretation that entails (the postulation of) the fewest possible entities in the domain of discourse. Second, the human parser is claimed to be guided by a Minimal Commitment strategy, in that it has a built-in preference for weak readings of privative ambiguities, which is purely a truth-conditional property. Third, Crain et al. argued for a difference between the adult parser and the language acquisition device, which is claimed to follow a Maximal Commitment strategy instead.

It is interesting to note that the evidence reported by Crain et al. for a Minimal Commitment strategy in adults is at odds with other hypotheses about adults' preferences in solving other kinds of privative ambiguities, like the Strongest Meaning Hypothesis or the Principle of Pragmatic Strengthening cited earlier. Despite their different orientation, though, all these hypotheses share the assumption that truth-conditional considerations are used in the resolution of semantic ambiguity. According to this view, the system would be blind to anything that doesn't specifically bear upon the entailment relations among alternative interpretations (like, for example, the properties of the extra-linguistic/visual context) while it would be affected by the semantic features of the linguistic input (like, for example, Downward Entailingness, that reverts the entailment pattern between alternatives). Indeed, different experimental studies have investigated the effect of the polarity of the linguistic environment on the interpretation of other linguistic phenomena such as Scalar Implicatures and, consistently with this hypothesis, found that these inferences were suspended in Downward Entailing contexts while they normally arose in Upward Entailing contexts, at least in adults (Chierchia et al., 2001, 2004; Noveck et al., 2002).

In this paper we concentrate on the study by Crain et al. In the next section, we replicate Crain et al.'s second experiment by manipulating a crucial component in the input, i.e. the availability of alternative referents in the context. Our approach is motivated by previous studies on the role of context in ambiguity resolution. For example, it has long been known that garden path effects like the one associated with the main-clause analysis of the sentence "The horse raced past the barn fell" (Bever, 1970; Frazier, 1979; Kimball, 1973; i.a.), that might be ultimately motivated by the Principle of Parsimony, get mitigated when the pragmatic/visual context is modified so as to include two horses (Crain and Steedman, 1985, see also Altmann et al., 1998; Altmann and Steedman, 1988; i.a.). In terms of the Principle of Parsimony, the reduced relative clause analysis is blocked in Bever's classic example because the parser, being parsimonious, does not postulate additional horses in the discourse domain and interprets "the horse" as the subject of a main clause. However, the reduced relative clause analysis is more easily accessed when the sentence is uttered in a context in which two (or more) horses are mentioned, because the presence of a plausible referent makes a relative clause construction easier to process.

Having introduced the Principle of Parsimony, our ultimate goal is to disentangle this principle and the Minimal Commitment strategy in the resolution of privative ambiguities, and we will do so by manipulating the context, as we will detail in Section 2. The implications of our findings extend beyond the particular constructions we will consider. While the Principle of Parsimony might be integrated within modular and interactive accounts of sentence processing, the Principle of Minimal Commitment requires a strictly modular view of the adult parser. In particular, according to this principle the semantic properties of the linguistic input (like, e.g., information about the entailing relation between alternatives in case of semantic ambiguity) are computed by a module that is specifically equipped to deal with semantic features and, as such, blind to external interferences.

By contrast, the Principle of Parsimony can easily account for the interference of extra-linguistic factors in the processing of ambiguous sentences. According to the Principle of Parsimony, adults should avoid postulating the existence of entities that were not previously mentioned. Thus, the application of this principle needs to take contextual information into consideration.

\section{Teasing strategies apart}

In discussing their experimental findings, Crain and colleagues proposed two separate claims about the human sentence processor. As we said, they claimed that the parser: (i) is parsimonious and thereby it avoids postulating the existence of entities that were not previously mentioned in the discourse; and (ii) it relies on a strategy of Minimal 
Commitment, favouring the weak interpretation in cases of privative ambiguity, i.e., one that has the most chances of being true. We argue that the specific contribution of each of these principles can be teased apart experimentally. Crain et al. tested sentences like (1) and claimed that adults preferred the anaphoric interpretation, because they falsified the sentences by imagining a context in which another individual of the same individual-level category of the subject DP was performing the action expressed by the verb. This finding has been interpreted by the authors as an indication that adults were using a Minimal Commitment strategy. However, we believe that this kind of response is compatible both with a Minimal Commitment strategy and a Parsimony strategy: upon encountering a sentence like (1), one might interpret it anaphorically either (i) because the anaphoric interpretation postulates a less rich context, i.e. a context that includes only elephants or (ii) because it is true in a wider set of circumstances.

To disentangle the contribution of the Principle of Parsimony and the contribution of the Minimal Commitment strategy, we conducted an experimental study structured along the lines of the original Crain et al. study, but with a crucial difference. In particular, we tested adults' preference for ambiguity resolution in response to different degrees of contextual information while leaving the truth-conditional properties of the alternative readings unchanged. Crucially, if Minimal Commitment is at play, adding contextual information should not affect the resolution of semantic ambiguity: the presence of an explicit context in which alternative referents for one are evoked or mentioned in the context should not lead subjects to shift to the exophoric reading, because the informativeness of the context does not affect the entailment pattern between the two interpretations of the ambiguous expression upon which the Minimal Commitment strategy relies. As a consequence, if the adult parser is guided by a Minimal Commitment strategy, as argued by Crain et al., the anaphoric interpretation of "the only one" should be favoured independently of the richness of the scenario, simply because this remains the weak reading. By contrast, if the resolution of privative ambiguities is guided by the Principle of Parsimony, an effect of context could emerge. In particular, it is conceivable that a richer context might lead adults to exploit all the entities introduced by the context and interpret "the only one" exophorically.

\subsection{Participants}

A total of 113 students of the University of Milano - Bicocca participated in this experiment. All participants were native speakers of Italian and were naïve with respect to the goals of the experiment. They were randomly assigned to 1 of the 3 conditions described in Section 2.2.1.

\subsection{Materials and methods}

\subsubsection{Materials}

We used a sentence falsification task in the form of a written questionnaire similar to the one used by Crain et al. (1994). Participants were given a set of 25 sentences. They were asked to read them silently ${ }^{1}$ and to provide a scenario in which these sentences were false. The sentences were presented one by one on a paper sheet, and nothing in the text was marked in bold or underlined or capitalized. Overall, 12 were critical ambiguous sentences containing the expression "l'unico" [lit. the only(one)] and the rest were fillers (see Appendix for a complete list of the stimuli used). For example, one of the target sentences used in our study was (3):

(3) La cocorita arancione è l'unica che si nasconde.

[the parakeet orange is the only that itself hides]

The orange parakeet is the only one that is hiding itself.

Three different conditions were created, in which the richness of the context varied between subjects to avoid an evident disparity in the length of the stimuli across conditions, while the target sentence to be falsified remained the same across conditions. For the first group of participants the target sentences were presented in isolation (no-context condition). For the second group of participants the target sentences were preceded by a short incipit mentioning a certain location like, e.g., "in the aviary" or "in the circus" (poor-context condition). Finally, participants belonging to the third group saw the target sentences preceded by a richer context in which, besides the indication of a certain location, other individuals were

${ }^{1}$ As one of the reviewers pointed out, prosody might play a role in the disambiguation process. We acknowledge that implicit prosody is always Q3 assigned in reading (Fodor, 2002). However, it is crucial to note that the experimental sentences in Italian remain ambiguous independently of prosody. In fact, the adjective appears in a post nominal position, to the right of the noun, and, in Italian, the prominence is assigned by default to the right at all levels of the hierarchal prosody (from sentence level to phrase level). In the case of the sentences used in our study, within the constituent or phonological phrase [N Adj], the relative prominence would be assigned to the element on the right by default, i.e. the adjective (Bocci, 2013; Nespor and Vogel, 1986). As a consequence, differently from what might happen in English, the sentence remains ambiguous in Italian, independently of the prosody implicitly or explicitly assigned to it. 
also introduced in the domain of discourse (rich-context condition). The context manipulation is exemplified in the schema below:

\section{no-context}

The orange parakeet is the only one that is hiding itself.

\section{poor-context}

In the aviary, the orange parakeet is the only one that is hiding itself.

\section{rich-context}

In the aviary, there are parakeets, parrots and canaries. The orange parakeet is the only one that is hiding itself.

The critical target sentences all contained a common noun that was accompanied by an adjective (e.g. orange parakeet), as in the original Crain et al. study. ${ }^{2}$ In order to allow more variability in the test material, we varied the superordinate category of the referent head noun so that the subject of the sentence could be a person, an animal or an inanimate object/thing. Furthermore, we varied the type of adjective that accompanied the referent noun: we used scalar (e.g. big) and colour adjectives (e.g. black). Let us briefly explain the reasoning behind this manipulation. The use of different kinds of adjectives was inspired by eye-tracking studies in which scalar and colour adjectives were contrasted in the exploration of a visual scene: anticipatory eye-movements towards a target referent were shown for scalar but not for colour adjectives (Sedivy, 2003, 2005; Sedivy et al., 1999). In particular, Sedivy et al. (1999) demonstrated that the presence of a discourse-available contrasting object (e.g. a short vs. a tall glass) exerted an effect on the on-line processing of referential expressions containing pre-nominal adjectives: i.e., adults could anticipate the target referent (e.g. the tall glass) at a point in which the input was still ambiguous. ${ }^{3}$ Following this line of research, one possibility is that one might more easily be interpreted by making reference to an individual belonging to the same individual-level category of the referent head noun when the adjective is scalar. By their nature (cf. Kennedy, 2007 i.a.), scalar adjectives require a contrast set to be interpreted and such a contrast set might be more easily identified in the individual-level category of the referent head noun. According to this hypothesis, the adjective big in (1) would be more naturally interpreted with respect to the class of elephants, thus favouring a substitution for one that taps on the individual-level category (elephants) and not the superordinate-level category (animals/entities in the context). Since that the complete set of the materials used by Crain et al. was not available for a comparison, we could not check if they varied the adjectives used in their experimental sentences or how they did so. Therefore, in our study we controlled for the effect of adjectival type in the process of domain restriction in the interpretation of the anaphora, varying the adjective type (scalar vs. non-scalar) within-subjects.

Finally, to control for a possible bias for associating the predicate and the subject DP used in our sentences (e.g. "parakeet" and "hiding oneself") we asked another group of 20 naïve subjects to complete a list that included the sentences to be used in the experiment, in which the simple subject DP was followed by suspension dots. The participants were instructed to fill in the dots with three verbs of their choice. For example, they received the fragment "The orange parakeet. .." and were asked to continue the sentence by mentioning the first three predicates that came to their mind and to write them down in the exact order in which that happened. We manually coded the responses provided and, in our experiment, we only used those predicates that were provided by the subjects in less than $3 \%$ of the cases as their first choice (and less than 7\% overall). With this, we meant to control for a possible bias towards the anaphoric interpretation due to the particular verb that has been used in the target sentences and prevent that subjects interpreted one in (3) as referred to "parakeets" simply because the predicate (hiding oneself) strongly led them towards this choice.

\subsubsection{Procedure and scoring}

As in the original study by Crain et al., participants were administered a written questionnaire. They were given a small booklet of 13 sheets. Each sheet in the booklet contained two sentences, each followed by a blank space. Participants were instructed to use the blank space below each sentence to write down a context that made that sentence false. Item position was pseudo-randomized in order to avoid consecutive trials of the same type. Subjects were instructed along the following lines:

\footnotetext{
${ }^{2}$ The presence of the adjective was necessary to allow for the anaphoric interpretation of the only one in a sentence in which a definite description (e.g. the parakeet) was used: without the adjective, sentence (3) would only allow for the exophoric interpretation, since the use of a definite description like the parakeet presupposes the presence of a unique parakeet in the domain of discourse, thus ruling the anaphoric interpretation out.

${ }^{3}$ Crucially, though, this facilitatory effect showed up only when the adjective denoted a property that is typically unlikely to be produced in describing the target object in isolation, such as material and scalar properties. In fact, no anticipatory eye-movements were recorded for properties that are frequently redundantly encoded, such as colour adjectives, even in presence of a discourse contrast (e.g. a blue vs. a red cup).
}

Please cite this article in press as: Foppolo, F., et al., The only one strategy in context. Lingua (2014), http://dx.doi.org/ 10.1016/j.lingua.2014.04.003 
(4) "In the booklet you will find a series of sentences. You have to imagine a situation that makes each of these sentences false. For example, suppose you were given the sentence "Donald Duck gave a bicycle to Goofy" and were asked to make it false. You have many ways to do so. For example, you could say that Donald Duck gave a bicycle to Mickey or that Donald Duck gave a car to Goofy orelse that Donald Duck gave a motorbike and a car to Goofy and so on. To fulfil this task you can choose the strategy you like the most, provided that the sentence is false in the context you come up with."

Given that participants were free to imagine their preferred scenario (as opposed to selecting one out of a range of possibilities offered by the experimenter) we obtained a wide range of responses. Upon inspection of the data, we first excluded all the responses that were completely irrelevant to the purposes of this study. For example, we excluded responses like (6) or (7) that were provided to falsify the target sentence in (5):

(5) TARGET: The orange parakeet is the only one that is hiding itself.

(6) All the birds flied off.

(7) There are no orange parakeets in the cage.

For the remaining responses, we decided to adopt a strict criterion for classification: we scored only the answers in which the critical expression "the only one" was explicitly falsified in the scenario provided by the participants, i.e. when the participant explicitly mentioned that another individual was performing the relevant action too (in addition to the character mentioned in the target sentence). Following this criterion, in response to the target sentence exemplified in (5), we included for the analyses only responses like (8), while we excluded responses like (9) that did not contain any explicit reference to the presupposition triggered by the use of only in the target sentence:

(8) (Not only the orange parakeet but) the green parakeet is hiding itself too.

(9) (Not the orange parakeet but) the green parakeet is hiding itself.

Once this first selection process was completed, participants' responses like (8) were scored with respect to the interpretation used in falsifying the target sentence: when an individual of the same individual-level category of the referent head noun was mentioned to falsify the sentence (as in (10)), the response was labelled anaphoric (AN); when an individual belonging to the superordinate category (but of a different individual-level type) was mentioned (as in (11)), the response was labelled exophoric (EX):

(10) The green parakeet is hiding itself too. [AN]

(11) The canary is hiding itself too. [EX]

\subsection{Results}

Once we eliminated the 72 missing or indecipherable answers ( $0.05 \%$ of the total), a total of 1284 responses remained. Of these, 431 were completely irrelevant to the purposes of this study and were not considered for the analyses (these were cases like (6) or (7)); 531 were responses like (9) and were not entered in the data analysis. Of the 322 responses like (8) selected by means of the criterion defined above, 204 (63\%) were anaphoric (AN) and $118(37 \%)$ were exophoric (EX). The distribution of the two types of answers across conditions is summarized in Table 1, while Fig. 1 plots the proportion of EX-responses as a function of Context (no vs. poor vs. rich):

As it is evident from the graph above, a difference was found in the type of responses provided in the 3 conditions, between the no-context and the rich-context conditions especially. In particular, the percentage of responses that are not compatible with the Minimal Commitment strategy (i.e. EX responses) is around $25 \%$ in the no-context condition but it rises to $65 \%$ in the rich-context condition.

Data were analyzed using mixed-effects analyses (Baayen et al., 2008) with random intercepts for subjects, nouncategory, and (nested in noun-category) items. A logistic mixed model (Jaeger, 2008) was adopted, taking the

Table 1

Distribution of type of answers (anaphoric vs. exophoric) across conditions.

\begin{tabular}{lllr}
\hline Condition & Anaphoric & Exophoric & Total \\
\hline No-context & 92 & 29 & 121 \\
Poor-context & 80 & 30 & 110 \\
Rich-context & 32 & 59 & 91 \\
\hline
\end{tabular}

Please cite this article in press as: Foppolo, F., et al., The only one strategy in context. Lingua (2014), http://dx.doi.org/ 10.1016/j.lingua.2014.04.003 


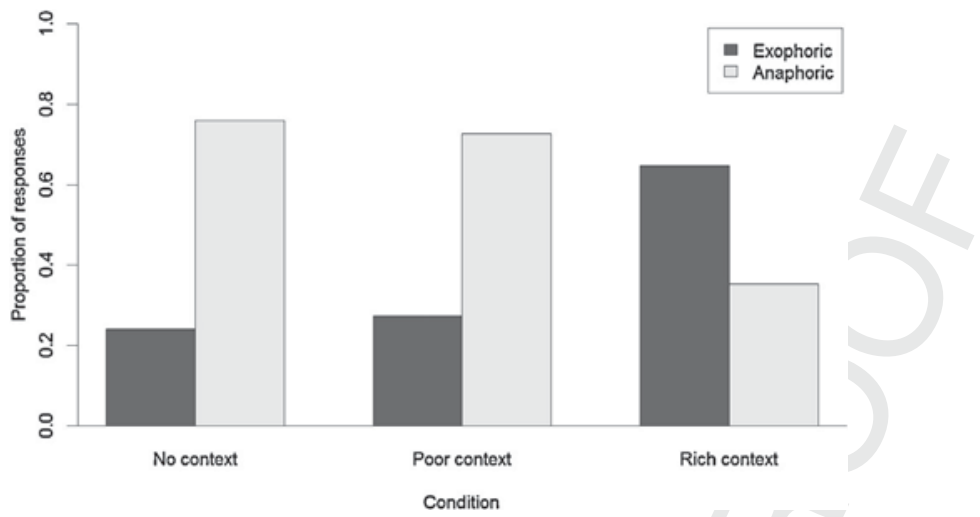

Fig. 1. Proportion of exophoric responses across conditions.

Table 2

Fixed effects in the logistic mixed model.

\begin{tabular}{lcrr}
\hline & Estimate & Std. error & $z$-Value \\
\hline Intercept & -1.62 & .52 & 3.09 \\
Poor-context & -.39 & .59 & .66 \\
Rich-context & -2.67 & .62 & .0019 \\
Adjective: scalar & -.35 & .52 & .34 \\
Adjective: scalar $\times$ poor-context & .01 & .69 & .68 \\
Adjective: scalar $\times$ rich-context & .53 & .70 & .001 \\
\hline
\end{tabular}

participants' answer (dichotomically coded, as described above) as the dependent variable. In other words, the likelihood of imagining a scenario that was compatible with an anaphoric interpretation was tested. The effects of two fixed variables were introduced in these models: the amount of context preceding the sentence, that varied across conditions (taking the no-context condition as the reference level) and the adjective type (taking colour adjectives as the reference level), as well as their interaction. Table 2 summarizes the fixed effects of the model, which was run on 322 data points. The random intercepts of subjects $(s d=1.11)$, item $(s d=.22)$ and noun-category $(s d=.47)$ were also included in the final model, accounting for the variance across participants, items and category of the nouns used. Random slopes were also tested, but their introduction did not significantly improve the overall goodness-of-fit (i.e., the chi-square test comparing the model before and after having introduced each random slope was not significant) and thus these were not considered in the final analysis, summarized in Table $2 .^{4}$

The analysis shows that participants were more likely to adopt an exophoric interpretation when a rich context was provided: i.e., they were more likely to mention an individual of the superordinate-level category when examples of these individuals were provided in the context that preceded the critical sentence. The difference between the no-context and the poor-context conditions was not significant. The parameters associated to adjective type and the interaction terms were not significant either.

\section{Final discussion}

Our results are consistent with some of the findings documented by Crain and colleagues. In the no-context condition adults preferred to falsify the target sentence selecting the anaphoric interpretation of "the only one," as in the original study by Crain et al. This finding is consistent with previous studies of garden-path phenomena (see e.g. Garnsey et al., 1997; Hare et al., 2007; McRae et al., 2005; Wilson and Garnsey, 2009): when the sentence is given in isolation, the parser resists postulating unnecessary entities, following the Principle of Parsimony.

With the intent of evaluating Crain et al.'s conflation of Parsimony and Minimal Commitment, we added different amounts of information to the context, so as to test how an enriched context affected the parsing of privative ambiguities. Contra Crain et al., we found that, when a rich context was provided, adults' preference for the anaphoric

\footnotetext{
${ }^{4}$ Equivalent results in the significance tests were obtained when a maximal random effect structure was introduced in the statistical model.
} 
interpretation of "the only one" gave way to the exophoric interpretation of the ambiguous expression. Indeed, adults exploited the information provided in the context and used it to solve the ambiguity. The fact that adults' preference for the weak reading dropped when a context was added does not rule out Parsimony, but it does cast doubts on the claim that the adult parser follows a strategy of Minimal Commitment to solve semantic ambiguity. This does not rule out the possibility that considerations about entailment matter for processes like domain restriction, but it certainly casts doubts on the theoretical explanations based solely on truth-conditional considerations like the Principle of Pragmatic Strengthening or the Strongest Meaning Hypothesis independently proposed to account for similar phenomena.

Our results are all the more important if we consider some of the consequences of Crain et al.'s proposal for the process of language acquisition. By comparing adults' and children's behaviour with respect to the phenomenon of one-substitution, these authors concluded that adults obeyed a Minimal Commitment strategy, preferring the weak interpretation in the resolution of the ambiguity, while children conformed to a strategy of Maximal Commitment, preferring the strong interpretation instead. On the basis of this difference, they argued for a difference between the characteristics that guide the adult parser and the mechanisms that operate in the Language Acquisition Device (LAD). On the basis of our results, however, we are not convinced that such a distinction is to be maintained in the first place. In fact, the tasks used for testing children and adults in the Crain et al. study were different. While adults were asked to falsify sentences in isolation, children were asked to judge sentences in a scenario in which individuals that did not belong to the same individual-level category of the referent for the head noun of the subject DP were present. This is similar to the no-context condition of the study presented here, in which subjects behaved as the adults in the original Crain et al.'s study, opting for the anaphoric (weak) interpretation. However, the adults in our rich-context condition behaved as the children in the original Crain et al.'s study, opting for the exophoric (strong) reading. Looking at Crain et al.'s results from this perspective, we do not see any strong argument in support of the distinction between the principles that guide the adult parser and the Language Acquisition Device advocated by those authors.

To conclude, although nothing can be said about the on-line mechanisms that underlie the processing of semantic ambiguity on the basis of this off-line study, we believe that our findings can inform future research on the resolution of privative ambiguities. Our study shows that context affects the disambiguation process and the process of domain restriction, so that the exophoric interpretation is more likely when a rich context is provided: participants tended to mention more entities of the superordinate category level when these entities had been previously mentioned in the context. In terms of general processing principles, we propose the following generalizations as a starting point for future investigation: (i) when no context is provided, adults follow a general Principle of Parsimony and do not extend the context by postulating additional entities, as independently argued for the resolution of temporarily ambiguous sentences; (ii) when the sentences are not given in isolation, however, adults follow a different strategy, that we might call Maximal Exploitation: when a context is provided, adults exploit it. In the case at hand, in the rich-context condition different entities were mentioned in the preceding discourse: we found that readers used these elements to interpret the linguistic input that followed. We speculate that they do so because they are following a very general strategy that urges them to exploit all the cues available in the context. While Parsimony readily explains the anaphoric preference found in the no- and poor-context conditions, the strategy of Maximal Exploitation that we propose accounts for the exophoric preference observed in the rich-context condition.

Our approach may also account for previous studies showing that context takes precedence over truth conditional considerations in the resolution of privative ambiguities in general. For example, the strategy of Maximal Exploitation Q4 might also explain the exact/at-least alternation in the interpretation of scalars in the paradigmatic example proposed by Welker, 1994; see also Carston, 1998), in which the numeral receives the "exact" interpretation in B's answer and the "at-least" interpretation in C's answer to A's utterance in (12):

A: I'm having a dinner party and I need four more chairs.

B: John has two chairs (exactly two, not more).

C: John has four chairs (at least four, eventually more).

In these examples, truth-conditional considerations about Informativeness that would favour the exact interpretation are overridden by considerations about contextual Relevance, so that the lower bound interpretation of the number term is accessed. The selection of the weaker meaning poses some problems to principles like the Principle of Pragmatic Strengthening, the Strongest Meaning Hypotheses or the Minimal Commitment strategy, for which truth-conditional considerations takes precedence in ambiguity resolution. By contrast, the principle of Maximal Exploitation proposed here might be applied to Welker's example and extended to other cases of ambiguity resolution or domain restriction as well, as it predicts that context takes precedence over truth-conditional considerations.

It remains a question for future research to extend the findings obtained here to a broader range of privative ambiguities, by modulating the weight of extra-linguistic cues and measuring their effects on the interpretation of the linguistic input. 


\section{Q5 Uncited references}

Bock and Warren (1985), Crain (1980), Crain and Thornton (1998), Frazier and Clifton (1997), R Development Core Team (2011), and Trueswell and Tanenhaus (1994).

\section{Acknowledgments}

This work was (partially) supported by funds from the European Science Foundation (Collaborative Research in Experimental Pragmatic, EURO-XPRAG) to the first author and by a VIDI grant from the Netherlands Organization for Scientific Research (NWO) to Andrea Gualmini. Special thanks go to Giuliano Bocci and Patrice Rusconi for insightful discussions.

\section{Appendix}

List of the critical sentences used in the three conditions, as detailed in the schema in Section 2.2.1.

1. [Nel negozio di animali [ci sono gattini, cagnolini e criceti.]] II gattino nero è l'unico che dorme. [In the pet shop [there are kittens, puppies and hamsters.] The black kitten is the only one that is sleeping.

2. [Alla fattoria [ci sono cavalli, pecore e mucche.] II cavallo bianco è l'unico che si lascia accarezzare. [In the farm [there are horses, sheep and cows.]l The white horse is the only one that let itself being pet.

3. [Nella voliera [ci sono cocorite, pappagalli e canarini.]] La cocorita arancione è l'unica che si nasconde. In the aviary [there are parakeets, parrots and canaries.]] The orange parakeet is the only one that is hiding itself.

4. [Al circo [ci sono trapezisti, clowns e domatori.]] II trapezista biondo è l'unico che ha fatto un inchino. [In the circus [there are aerialists, clowns and tamers.]] The blonde aerialist is the only one that bowed.

5. [Nel centro di accoglienza [ci sono tunisini, marocchini ed egiziani.]] II ragazzo tunisino è l'unico che è stato soccorso. [In the immigrant shelter [there are Tunisians, Moroccans and Egyptians.]] The Tunisian boy is the only one that has been assisted.

6. [Nella composizione [ci sono gigli, rose e tulipani.]] II giglio bianco è li'unico che è appassito. [In the composition [there are lilies, roses and tulips.]] The white lily is the only one that withered.

7. [Nella pozza [ci sono ippopotami, elefanti e giraffe.]] L'ippopotamo piccolo è l'unico che fa il bagno. [In the pond [there are hippos, elephants and giraffes.]] The small hyppo is the only one that is taking a bath.

8. [Nella giungla [ci sono scimmie, tigri e serpenti.]] La scimmia grande è l'unica che è stata catturata. [In the jungle [there are monkeys, tigers and snakes.l] The big monkey is the only one that has been captured.

9. [Alla festa della ditta [ci sono segretarie, operai e dirigenti.]] La segretaria bella è l'unica che danza. [At the firm party [there are secretaries, workmen and managers.]] The beautiful secretary is the only one that is dancing.

10. [Nel quartiere di Ginza [ci sono villette, palazzine e grattacieli.]] La palazzina bassa è l'unica che è scampata al terremoto. [In the suburb of Ginza [there are houses, blocks of flats and skyscrapers]] The small house is the only one that survived the earthquake.

11. [Nel bosco [ci sono pini, larici e abeti.]] II pino alto è l'unico che è stato abbattuto. [In the wood there pines, larches and firs.]] The tall pine is the only one that has been torn down.

12. [Nella radura [ci sono gazzelle, bufali e zebre.]] La gazzella veloce è l'unica che si è salvata. [In the glade [there are gazelles, buffalos and zebras.J] The fast gazelle is the only one that saved itself.

\section{References}

Altmann, G., Steedman, M., 1988. Interaction with context during human sentence processing. Cognition 30, 191-238.

Altmann, G., van Nice, K., Garnham, Y., Henstra, A.J.A., 1998. Late closure in context. J. Mem. Lang. 38, $459-484$.

Baayen, R.H., Davidson, D.J., Bates, D.M., 2008. Mixed effects modeling with crossed random effects for subjects and items. J. Mem. Lang. 59, 390-412.

Bever, T.G., 1970. The cognitive basis for linguistic structures. In: Hayes, J.R. (Ed.), Cognition and the Development of Language. Wiley, New York.

Bocci, G., 2013. The Syntax-Prosody Interface: A Cartographic Perspective with Evidence from Italian, vol. 204. John Benjamins Publishing. Bock, J.K., Warren, R.K., 1985. Conceptual accessibility and syntactic structure in sentence formulation. Cognition 21, 47-67.

Carston, R., 1998. Informativeness, relevance and scalar implicature. In: Carston, R., Uchida, S. (Eds.), Relevance Theory: Applications and Implications, pp. 179-236

Chierchia, G., 2006. Broaden your views: implicatures of domain widening and the "Logicality" of language. Ling. Inq. 37 (4), $535-590$. 
Chierchia, G., Crain, S., Guasti, M.T., Gualmini, A., Meroni, L., 2001. The acquisition of disjunction: evidence for a grammatical view of scalar implicatures. In: Do, A.H.-J., Domínguez, L., Johansen, A. (Eds.), BUCLD 25 Proceedings. Cascadilla Press, Somerville, MA, pp. 157-168.

Chierchia, G., Guasti, M.T., Gualmini, A., Meroni, L., Crain, S., Foppolo, F., 2004. Semantic and pragmatic competence in children's and adults' comprehension of OR. In: Noveck, I.A., Sperber, D. (Eds.), Experimental Pragmatics. Palgrave Macmillan, New York, NY, pp. $283-300$.

Chierchia, G., Fox, D., Spector, B., 2013. The grammatical view of scalar implicatures and the relationship between semantics and pragmatics. In: Portner, P., Maienborn, C., von Heusinger, K. (Eds.), Handbook of Semantics. Mouton de Gruyter, Berlin.

Crain, S., 1980. Pragmatic Constraints on Sentence Comprehension. (Unpublished Ph.D. dissertation) University of California, Irvine.

Crain, S., 1992. The semantic subset principle in the acquisition of quantification. In: Paper Presented at the Workshop on the Acquisition of Wh-Extraction and Related Work on Quantification. University of Massachusetts, Amherst.

Crain, S., 1993. Semantic substitutions. In: Invited Paper Presented at the Center for Cognitive Science Conference: Early Cognition and the Transition to Language. University of Texas, Austin.

Crain, S., McKee, C., 1985. The acquisition of structural restrictions on anaphora. In: Proceedings of NELS 15. GLSA. University of Massachusetts, Amherst, pp. 94-110.

Crain, S., Philip, W., 1993. Global semantic dependencies in child language. In: Paper Presented at the 16th GLOW Colloquium, Lund, Sweden. Crain, S., Steedman, M., 1985. On not being led up the garden path: the use of context by the psychological parser. In: Dowty, D., Karttunnen, L. Zwicky, A. (Eds.), Natural Language Parsing. Cambridge University Press, Cambridge, UK.

Crain, S., Thornton, R., 1998. Investigations in Universal Grammar. MIT Press, Cambridge, MA.

Crain, S., Ni, W., Conway, L., 1994. Learning, parsing and modularity. In: Clifton, C., Frazier, L., Rayner, K. (Eds.), Perspectives on Sentence Processing. LEA, Hillsdale, NJ.

Dalrymple, M., Kanazawa, M., Kim, Y., Mchombo, S., Peters, S., 1998. Reciprocal expressions and the concept of reciprocity. Ling. Philos. 159-210.

Frazier, L., 1979. On Comprehending Sentences: Syntactic Parsing Strategies. (Ph.D. dissertation) University of Connecticut, Indiana University Linguistics Club.

Frazier, L., Clifton, C., 1997. Construal: overview, motivation, and some new evidence. J. Psycholing. Res. 263, $277-295$.

Garnsey, S.M., Pearlmutter, N.J., Myers, E., Lotocky, M.A., 1997. The contributions of verb bias and plausibility to the comprehension of temporarily ambiguous sentences. J. Mem. Lang. 37, 58-93.

Geurts, B., 2000. Buoyancy and strength. J. Semant. 17, 315-333.

Gualmini, A., Schwarz, B., 2009. Solving learnability problems in the acquisition of semantics. J. Semant. 26, 185-215.

Gualmini, A., Hulsey, S., Hacquard, V., Fox, D., 2008. The question-answer requirement for scope assignment. Nat. Lang. Semant. 16, 205-237.

Hare, M., Tanenhaus, M.K., McRae, K., 2007. Understanding and producing the reduced relative construction: evidence from ratings, editing and corpora. J. Mem. Lang. 56, 410-435.

Horn, L.R., 1989. A Natural History of Negation, vol. 960. University of Chicago Press, Chicago.

Jaeger, T.F., 2008. Categorical data analysis: away from ANOVAs (transformation or not) and towards logit mixed models. J. Mem. Lang. 59, 434-446.

Kennedy, C., 2007. Vagueness and grammar: the semantics of relative and absolute gradable adjectives. Ling. Philos. 30, 1-45.

Kimball, J., 1973. Seven principles of surface structure parsing in natural language. Cognition 2, 15-47.

Krifka, M., 1998. Pragmatic strengthening in plural predications and donkey sentences. In: Proceedings of the Eighth Conference on Semantics and Linguistic Theory. Ithaca. Cornell University.

Ladusaw, W.A., 1980. Polarity Sensitivity as Inherent Scope Relations. Indiana University Linguistics Club, Bloomington, Indiana

McRae, K., Hare, M., Tanenhaus, M.K., 2005. Meaning Through Syntax is insufficient to explain comprehension of sentences with reduced relative clauses: a critique of McKoon, Ratcliff 2003. Psychol. Rev. 112, 1022-1031.

Musolino, J., 2006. On the semantics of the subset principle. Lang. Learn. Dev. 23, 195-218.

Nespor, M., Vogel, I., 1986. Prosodic Phonology. Foris, Dordrecht.

Noveck, I., Chierchia, G., Chevaux, F., Guelmiuger, R., Sylvestre, E., 2002. Linguistic-pragmatic factors in interpreting disjunctions. Think. Reason. 8, 297-326.

R Development Core Team, 2011. R: A Language and Environment for Statistical Computing. R Foundation for Statistical Computing, Vienna, Austria, http://www.R-project.org

Sedivy, J.C., 2003. Pragmatic versus form-based accounts of referential contrast: evidence for effects of informativity expectations. J. Psycholing Res. 321, 3-23.

Sedivy, J.C., 2005. Evaluating explanations for referential context effects: evidence for Gricean mechanisms in online language interpretation: In: Trueswell, J.C., Tanenhaus, M.K. (Eds.), Approaches to World-Situated Language Use: Bridging the Language-as-product and Language-asaction Traditions Learning, Development, and Conceptual Change. MIT Press, Cambridge, MA, pp. 345-364.

Sedivy, J.C., Tanenhaus, M., Chambers, C., Carlson, G., 1999. Achieving incremental semantic interpretation through contextual representation. Cognition 71, 109-147.

Trueswell, J.C., Tanenhaus, M.K., 1994. Toward a lexicalist framework of constraint-based syntactic ambiguity resolution. In: Clifton, C., Rayner, K., Frazier, L. (Eds.), Perspectives on Sentence Processing. Hiilsdale, NJ, Erlbaum, pp. 155-179.

Welker, K., 1994. Plans in the Common Ground: Toward a Generative Account of Implicature. (Ph.D. dissertation) The Ohio State University, Columbus.

Wilson, M.P., Garnsey, S.M., 2009. Making simple sentences hard: verb bias effects in simple direct object sentences. J. Mem. Lang. 60, 368-392.

Winter, Y., 2001. Plural predication and the Strongest Meaning Hypothesis. J. Semant. 18, 333-365.

Zwicky, A.M., Sadock, J.M., 1975. Ambiguity tests and how to fail them. In: Kimball, J.P. (Ed.), Syntax and Semantics, 4. Academic Press, New York. 